\title{
On the Inadequacy of the CVR Factor for Active Schemes
}

DOI:

10.1109/TPWRD.2019.2944750

\section{Document Version}

Accepted author manuscript

Link to publication record in Manchester Research Explorer

\section{Citation for published version (APA):}

Gutierrez-Lagos, L., \& Ochoa, LN. (2019). On the Inadequacy of the CVR Factor for Active Schemes. I E E E Transactions on Power Systems. https://doi.org/10.1109/TPWRD.2019.2944750

\section{Published in:}

I E E E Transactions on Power Systems

\section{Citing this paper}

Please note that where the full-text provided on Manchester Research Explorer is the Author Accepted Manuscript or Proof version this may differ from the final Published version. If citing, it is advised that you check and use the publisher's definitive version.

\section{General rights}

Copyright and moral rights for the publications made accessible in the Research Explorer are retained by the authors and/or other copyright owners and it is a condition of accessing publications that users recognise and abide by the legal requirements associated with these rights.

\section{Takedown policy}

If you believe that this document breaches copyright please refer to the University of Manchester's Takedown Procedures [http://man.ac.uk/04Y6Bo] or contact uml.scholarlycommunications@manchester.ac.uk providing relevant details, so we can investigate your claim.

\section{OPEN ACCESS}




\title{
On the Inadequacy of the CVR Factor for Active Schemes
}

\author{
L. Gutierrez-Lagos, Graduate Student Member, IEEE and L. F. Ochoa, Senior Member, IEEE
}

\begin{abstract}
Conservation Voltage Reduction (CVR) is a wellknown technique to reduce energy consumption by reducing the supplied voltage within statutory limits. Historically, the efficacy of CVR schemes has been quantified using the CVR factor (CVRf) which relates, in a given period, voltage reduction to energy savings. This metric has proved useful in the past, where fixed voltage reductions were applied. However, this letter shows from a theoretical perspective and with a realistic case study that when used for active CVR schemes (voltages actively controlled throughout the day), the CVRf can be smaller than that for fixed voltage reductions despite the larger energy savings. This could be misleading for decision makers considering active CVR schemes as an energy efficiency measure. Consequently, it is recommended that comparisons among CVR schemes are carried out in terms of actual energy reductions.
\end{abstract}

Index Terms-Conservation voltage reduction, CVR factor, load models, voltage control.

\section{INTRODUCTION}

$\mathrm{C}_{\mathrm{s}}$ CONSERVATION Voltage Reduction (CVR) is a well-

known method to reduce energy consumption by reducing the supplied voltage within statutory limits. This method, investigated since the '70s in different countries [1], takes advantage of the positive correlation of voltage and demand featured by most appliances. To quantify its efficacy, the CVR factor $\left(C V R_{f}\right)$ has been typically adopted. This is defined as the ratio between the percentage energy savings obtained $(\Delta E)$ over the applied percentage voltage reduction $(\Delta V)$, as in (1).

$$
C V R_{f}=\frac{\Delta E}{\Delta V}
$$

Due to the historical lack of observability in distribution networks, CVR has been traditionally implemented by adopting modest, fixed voltage reductions at primary substations equipped with on-load tap changers (OLTCs) [1]. In this case, $\Delta V$ corresponds to a single value for the whole period (small variations can occur). More sophisticated (active) CVR schemes have included monitoring at critical points $[2,3]$ or even used smart meters to monitor customer voltages [3-5]. This has allowed increasing energy savings by actively determining adequate voltage reductions throughout the day. The

This work was supported in part by Electricity North West, U.K., through the Ofgem's Low Carbon Networks Fund Tier 2 Project "Smart Street," 2014-2017 and in part by the EPSRC project MY-STORE (Grant EP/N001974/1).

L. Gutierrez-Lagos is with the School of Electrical and Electronic Engineering, The University of Manchester, M13 9PL, UK and L. F. Ochoa is with the Department of Electrical and Electronic Engineering, The University of Melbourne, VIC 3010, Australia and with the School of Electrical and Electronic Engineering, The University of Manchester, M13 9PL, UK (e-mails: luis.gutierrez.1@ieee.org, luis_ochoa@ieee.org)

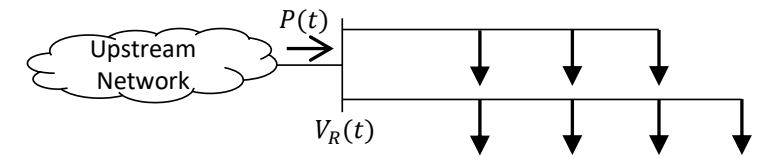

Fig. 1. Generic network

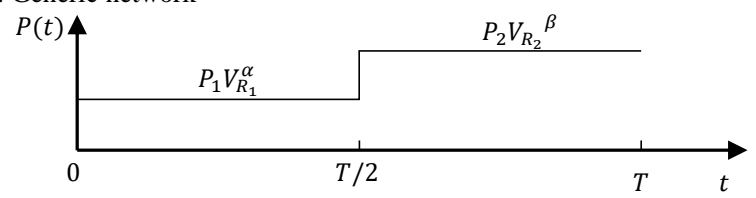

Fig. 2. Two-blocks 'load profile'

$C V R_{f}$ has also been used in these cases by considering the difference of voltage averages for the case without (business as usual, BAU) and with CVR, as shown in (2), where $T$ represents the sampling interval. For instance, this procedure was adopted in [3] for eleven American utilities.

$$
\Delta V=\frac{\sum_{t=1}^{T} V_{B A U}(t) / T-\sum_{t=1}^{T} V_{C V R}(t) / T}{\sum_{t=1}^{T} V_{B A U}(t) / T}
$$

This letter shows from a theoretical perspective and with a realistic case study that this practice can be misleading: the $C V R_{f}$ of active schemes can be smaller than that for fixed voltage reductions despite the larger energy savings.

\section{THE INADEQUACY OF THE $C V R_{f}$ FOR ACTIVE SCHEMES}

Let us consider the generic network shown in Fig. 1, where the voltage $V_{R}(t)$ can be regulated at time $t$. This could be, for instance, the busbar voltage at a primary substation equipped with OLTCs. The aggregated demand seen at the regulated bus corresponds to $P(t)$. For simplicity, let us consider two consecutive demand blocks for the aggregated load, both with a duration of $T / 2$. Although, in reality, customers within a network might have different voltage-demand characteristics at different times, let us assume that the aggregated load of each block can be represented with a single exponential model [6], with parameters $\alpha$ and $\beta$, respectively. This is illustrated in Fig. 2. $P_{1}$ and $P_{2}$ represent the aggregated active power at nominal voltage for blocks 1 and 2 , respectively. $V_{R_{1}}$ and $V_{R_{2}}$ are the regulated bus voltage values for each block.

The voltage reduction that can be applied at the regulated bus will depend on the loading condition and the corresponding voltage drops in the downstream network. Given the smaller demand in the first block $\left(P_{1}<P_{2}\right)$, the minimum voltage that can be applied will be lower (i.e., larger voltage reduction) than the one that can be applied in the second block. Let us denote $V_{1}$ and $V_{2}$ the minimum voltages that can be supplied without producing customer voltages below statutory limits for blocks 1 and 2, respectively $\left(V_{1}<V_{2}\right)$. 


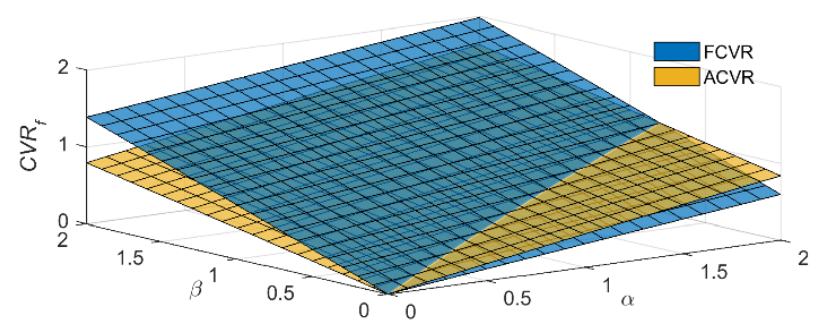

Fig. 3. CVR factors for both schemes as a function of the load parameters

Let us now define three cases: business as usual (BAU), fixed CVR (FCVR), and active CVR (ACVR). For the BAU case, the regulated voltage is kept fixed, $V_{R}(t)=V_{B A U}$ $\left(V_{B A U}>V_{2}\right)$. For the FCVR, $V_{R}(t)=V_{2}$ as it is the minimum fixed voltage that can be applied. Finally, for the ACVR, $V_{1}$ and $V_{2}$ are used in their respective blocks. The corresponding energy consumed for the BAU, FCVR, and ACVR cases are given by (3), (4), and (5), respectively.

$$
\begin{gathered}
E_{B A U}=P_{1} V_{B A U}^{\alpha} T / 2+P_{2} V_{B A U}^{\beta} T / 2 \\
E_{F C V R}=P_{1} V_{2}^{\alpha} T / 2+P_{2} V_{2}^{\beta} T / 2 \\
E_{A C V R}=P_{1} V_{1}^{\alpha} T / 2+P_{2} V_{2}^{\beta} T / 2
\end{gathered}
$$

Note that $\alpha$ and $\beta$ can take values between 0 and 2 (constant power and constant impedance, respectively), and that $V_{1}<V_{2}<V_{B A U}$. Therefore, $V_{1}^{\alpha}<V_{2}^{\alpha}$ and $E_{A C V R}<E_{F C V R}<E_{B A U}$. Consequently, the energy savings from the active CVR are larger than those from the fixed CVR, as expected. The CVR factors of each scheme are calculated with (6), where $E_{C V R}$ is replaced with $E_{A C V R}$ or $E_{F C V R}$ as corresponds, and the average voltage reduction is given by (7).

$$
\Delta V=\left\{\begin{array}{c}
\frac{C V R_{f}=}{\frac{\left(V_{B A U}-\left(V_{1}+V_{2}\right) / 2\right)}{V_{B A U}} \text { for } A C V R} \\
\frac{V_{B A U}-E_{2}}{V_{B A U}} \text { for FCVR }
\end{array}\right.
$$

Although the ACVR always delivers larger energy savings than the FCVR, its $C V R_{f}$ can be larger or smaller depending on the combinations of $\alpha$ and $\beta$, as well as powers and voltages. This is shown in Fig. 3 where $\alpha$ and $\beta$ can vary from 0 to 2 , and where the following parameters have been used: $V_{B A U}=1, V_{1}=0.95, V_{2}=0.98, P_{1}=0.3, P_{2}=0.7$, and $T=2$ (all in $\mathrm{pu}$ ). This selection aims to represent (in a simplified manner) the differences between low and high demand periods in a day (blocks 1 and 2, respectively).

In this example, the $C V R_{f}$ of the ACVR scheme is smaller than the one from the FCVR for most of the load model parameter combinations. Indeed, the opposite happens only after $\alpha$ is about 2.5 times larger than $\beta$; which is very unlikely in practice [8]. Indeed, low demand periods are likely to be dominated by fridges (between constant power and constant current), electronics such as phone chargers (constant power) and some lighting (mixed), resulting in $\alpha<1$ (e.g., early hours of a day). During high demand periods, the presence of more appliances (some with constant impedances) make $\beta$ to be larger (also shown in [8] for the UK). For instance, $\alpha=0.5$ and $\beta=1.2$ results in $E_{B A U}=1, E_{F C V R}=0.980$ and $E_{A C V R}=0.976$. Although both CVR schemes show similar values, the energy savings

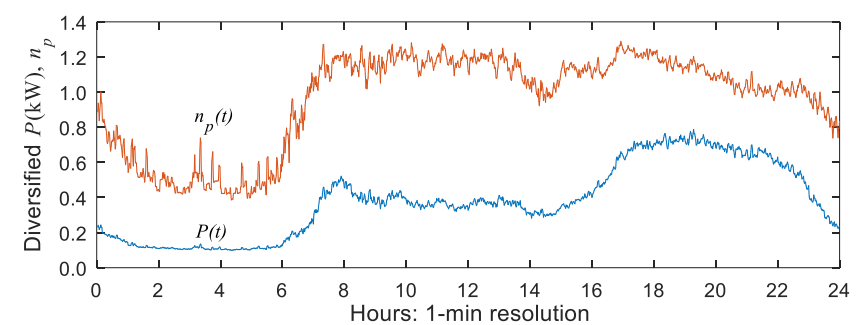

Fig. 4. Diversified load profile and model

from the ACVR scheme are larger, as expected. However, the CVR factors are 0.99 and 0.70 for the FCVR and ACVR schemes, respectively. This shows a large difference which mistakenly suggests that the active scheme performs worse than the fixed voltage reduction despite the (slightly) larger energy reduction. The above demonstrates, with a simple case, that the $C V R_{f}$ is inadequate to assess active CVR schemes.

\section{CASE STUDY}

The inadequacy of the $C V R_{f}$ is now shown in a real UK residential LV network where the two types of CVR schemes are assessed. For a more realistic calculation of energy consumption, instead of assuming an aggregated load model, this case study captures the time-varying nature of the network load by modelling individual LV customers (houses) in detail. This extends the preliminary analysis performed in [9].

\section{A. LV Network, Time-Varying Load Profiles and Models}

The adopted LV network supplies 268 single-phase customers through 3 underground four-wire feeders. The transformer has a typical UK ratio of $6.6 / 0.433 \mathrm{kV}(8.7 \%$ boost compared to the nominal voltage of $400 \mathrm{~V} \mathrm{~L}-\mathrm{L}$ ). It is equipped with an OLTC that regulates voltages between 0.95 and $1.1 \mathrm{pu}$ and has remote monitoring capabilities. Customers are modelled using a pool of 1-min resolution load profiles and models for a winter weekday. The resulting diversified demand $P(t)$ and exponential model parameter $n_{p}(t)$ are shown in Fig. 4, for illustration purposes. Further details are presented in [9].

\section{B. BAU and CVR Schemes}

The different schemes are implemented as follows:

- $\underline{B A U}:$ No voltage reductions applied. The source voltage is considered -for simplicity- fixed at $1 \mathrm{pu}$ and the transformer has a typical voltage boost of $8.7 \%$.

- FCVR: Considers a fixed voltage reduction of $4.29 \%$. This emulates a 3 tap position change in the primary substation.

- ACVR: The active CVR control for OLTC-enabled LV networks, proposed in [9], is implemented. This adjusts the voltage target at the LV busbar every control cycle (30-min in this study) to keep the lowest customer voltage (remote point) close to a reference ( $0.96 \mathrm{pu}$ in this study). The source voltage is considered fixed at $1 \mathrm{pu}$ as in the BAU case.

\section{Results}

Fig. 5 illustrates the performance of both schemes, where it is shown that the ACVR manages to keep the minimum customer voltage close to the reference. This allows for much larger voltage reductions throughout the day and, therefore, larger energy savings. Indeed, the ACVR reaches about $80 \%$ more network energy savings than the FCVR scheme ( $\Delta E$ of $8.29 \%$ and $4.65 \%$, respectively), demonstrating the advantage 


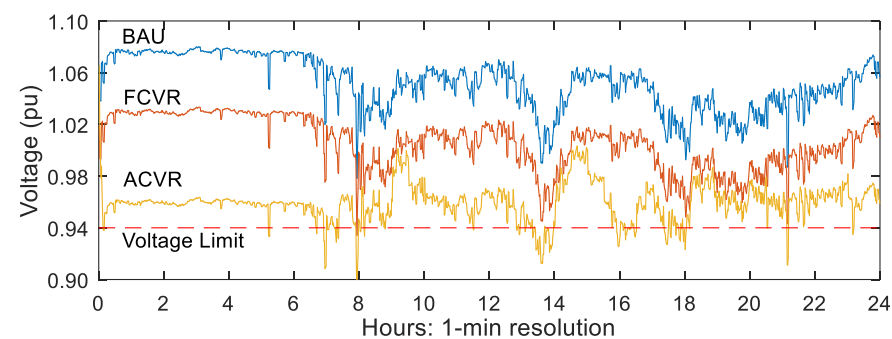

Fig. 5. Voltages at the remote point for the different schemes

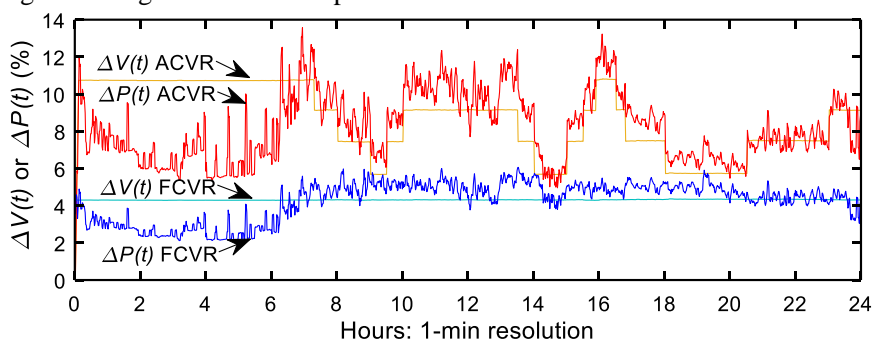

Fig. 6. Voltage and power reduction through time for both schemes

of such methods. However, its $C V R_{f}$ is only 0.96 compared to 1.08 for the FCVR. As mentioned earlier, this is due to the use of the daily average voltage reduction in the $C V R_{f}$ calculation, which is twice as big for the ACVR (8.65\%) compared to $4.31 \%$ for the FCVR. The issue raises as the ACVR reaches its largest voltage reduction during the early hours due to light loading (small voltage drops). This period, however, happens to be the least responsive, as shown in Fig. 4 (the reverse situation could occur with more electrical heating [10]). Thus, $\Delta V$ is significantly larger at early hours but without bringing proportional percentage power reductions, resulting in a smaller $C V R_{f}$. The latter can be seen in Fig. 6 where the obtained power reduction $\triangle P(t)$ is shown for both schemes (8).

$$
\Delta P(t)=\frac{P_{B A U}(t)-P_{C V R}(t)}{P_{B A U}(t)}
$$

From Fig. 6 is also clear that the shape of $\Delta P(t)$ for the FCVR follows that of the $n_{p}(t)$ shown in Fig. 4. Thus, for a fixed $\Delta V$, the obtained $\Delta P(t)$ has a nearly linear relationship with $n_{p}(t)$. This can be explained as follows: for small voltage variations, the discrete power to voltage change can be approximated by (9). Then, using an exponential model in pu and evaluating around the nominal voltage, (10) is obtained. Finally, the linear relation (for a fixed $\Delta V$ ) is derived in (11).

$$
\begin{gathered}
\Delta P \approx \frac{d P}{d V} \Delta V \\
\frac{d P}{d V}=n_{p} V^{n_{p}-1}=n_{p} \\
\Delta P \approx \frac{d P}{d V} \Delta V=n_{p} \Delta V
\end{gathered}
$$

This also means that, if the $C V R_{f}$ is calculated in terms of power and voltage changes through the day (as suggested in [6]), its value would be very close to $n_{p}(t)$, as shown in (12), regardless the scheme. This is verified in Fig. 7, where the $C V R_{f}(t)$ is plotted for both schemes together with the diversified load model of the customers, $n_{p}(t)$. While both $C V R_{f}(t)$ are very similar, $n_{p}(t)$ is slightly different as it does not incorporate losses (weighted average of each customer $n_{p}(t)$ ).

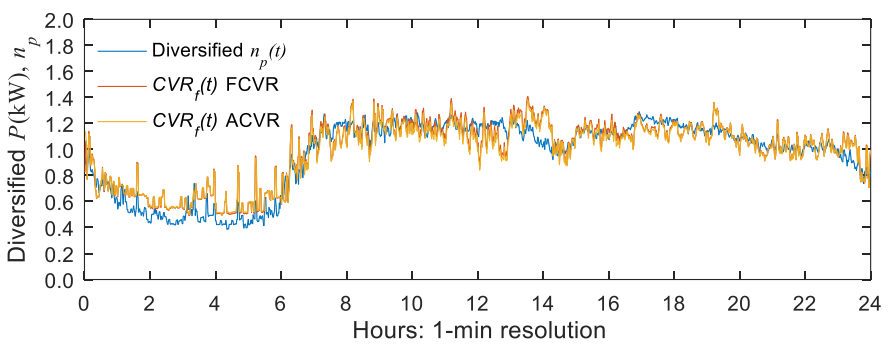

Fig. 7. $C V R_{f}(t)$ calculated in terms of instant power and voltage reductions

$$
C V R_{f}(t)=\frac{\Delta P(t)}{\Delta V(t)} \approx n_{p}(t)
$$

Although the $C V R_{f}(t)$ helps identifying the most responsive times to voltage changes, it does not help relating energy savings to voltage reductions across the window of interest. Moreover, these values, per time step or as an average, would be very similar regardless the CVR scheme; making it unsuitable for comparison purposes.

\section{CONCLUSIONS}

This letter provided theoretical and practical demonstrations of the inadequacy of the widely-used CVR factor $\left(C V R_{f}\right)$ to quantify energy savings for active schemes (voltages actively controlled). The issue comes from using the voltage reduction average and the fact that the voltage dependency of the demand varies in time. As periods have different voltage dependency, the different voltage reductions can result in different power reductions and, therefore, energy. This can result in smaller $C V R_{f}$ for active schemes compared to fixed reduction schemes, despite the larger energy savings achieved.

Moreover, if the $C V R_{f}$ is calculated each time step (power and voltage reductions), the result would be nearly equal to $n_{p}(t)$ regardless the scheme. This does not reflect differences in energy reduction, making it unsuitable for comparisons.

Consequently, it is recommended that comparisons among CVR schemes are done in terms of actual energy reductions.

\section{REFERENCES}

[1] W. Zhaoyu and W. Jianhui, "Review on Implementation and Assessment of Conservation Voltage Reduction," IEEE Trans. Power Syst., vol. 29, pp. 1306-1315, 2014.

[2] T. L. Wilson and D. G. Bell, "Energy conservation and demand control using distribution automation technologies," in Rural Electric Power Conference, 2004, 2004, pp. C4-1-12.

[3] K. Fagen and C. Bernier, "Efficiencies in distribution design and operating practices mid-study analysis (February 2007)," in Rural Electric Power Conference, 2007 IEEE, 2007, pp. B6-B6-14.

[4] M. A. Peskin, et al., "Conservation Voltage Reduction with feedback from Advanced Metering Infrastructure," in IEEE/PES Transm. and Distrib. Conf. and Exp. (T\&D), 2012, pp. 1-8.

[5] I. Roytelman and J. Medina, "Volt/VAR control and Conservation Voltage Reduction as a function of advanced DMS," in IEEE/PES Innovative Smart Grid Technologies Conference (ISGT), 2016, pp. 1-4.

[6] Z. Wang and J. Wang, "Time-Varying Stochastic Assessment of Conservation Voltage Reduction Based on Load Modeling," IEEE Trans. Power Syst., vol. 29, pp. 2321-2328, 2014.

[8] A. J. Collin, et al., "Development of low-voltage load models for the residential load sector," IEEE Trans. Power Syst., vol. 29, pp. 21802188, 2014.

[9] L. Gutierrez-Lagos, et al., "Unlocking CVR Benefits Using Active Voltage Control in LV Networks," in 2018 Power Systems Computation Conference (PSCC), Dublin, Ireland, 2018, pp. 1-7.

[10] L. Gutierrez-Lagos and L. F. Ochoa, "CVR assessment in UK residential LV networks considering customer types," in IEEE/PES Innovative Smart Grid Technologies - Asia (ISGT-Asia), 2016, pp. 1-6. 